University of Rhode Island

DigitalCommons@URI

Open Access Master's Theses

1980

\title{
Factors Influencing Four Rules for Determining the Number of Principal Components to Retain
}

William R. Zwick

University of Rhode Island

Follow this and additional works at: https://digitalcommons.uri.edu/theses

\section{Recommended Citation}

Zwick, William R., "Factors Influencing Four Rules for Determining the Number of Principal Components to Retain" (1980). Open Access Master's Theses. Paper 1577.

https://digitalcommons.uri.edu/theses/1577

This Thesis is brought to you for free and open access by DigitalCommons@URI. It has been accepted for inclusion in Open Access Master's Theses by an authorized administrator of DigitalCommons@URI. For more information, please contact digitalcommons-group@uri.edu. 
FACTORS INFLUENCING FOUR RULES FOR

DETERMINING THE NUMBER OF PRINCIPAL COMPONENTS TO RETAIN

BY

WILLIAM R. ZWICK

A THESIS SUBMITTED IN PARTIAL FULFILLMENT OF THE

REQUIREMENTS FOR THE DEGREE OF

MASTER OF ARTS

IN

PSYCHOLOGY

UNIVERSITY OF RHODE ISLAND

1980 


\section{ABSTRACT}

A common problem encountered in the applied use of principal components analysis (PCA) as a data reduction technique is the determination of the number of components to retain. This problem is particularly acute when some method of rotation is to be employed. This study examined the consequences of employing each of four decision methods upon PCA's of correlation matrices made up of systematically differing numbers of variables and subjects and based upon known systematically differing underlying component structures. Three levels of number of variables $(36,72,144)$, sample size $(75,150,450)$ and number of components $(3,6,12)$ were examined at each of two $(.50$ and .80) levels of component saturation. Within each example each component was defined by the same number of variables. No unique components nor complex variables were included in the set of computer generated data.

It was found the different rules lead to dramatically different results. In general, the scree test (SCREE) and the minimum average partial test (MAP) were the most accurate. The Bartlett test (BART) was somewhat less accurate and the eigenvalue greater than one rule (K1) was quite inaccurate. Increases in sample size and component saturation improved the performance of the rules. Increases in the number of variables examined increased the accuracy of SCREE and MAP, did not effect BART markedly but drasticly decreased KI's accuracy. KI often greatly overestimated the number of components to retain. 
Combinations of factors lead to the most inaccurate cases for each rule. K1 performed worst at low component saturation with high numbers of variables. MAP and SCREE performed worst at low component saturation with small samples, small numbers of variables and a large number of components. BART performed worst when the number of variables was almost equal to the sample size.

The single and interacting effects of the variables upon the rules is discussed. Guidelines for applied use are presented. Useful future research in this area is indicated. 


\section{ACKNOWLEDGEMENTS}

Thanks first to my conmittee whose thoughtful direction clarified the research questions early and often. Special gratitude must be extended to the University of Rhode Is land's Academic Computer Center. Their cooperation and support was as available and essential as their excellent equipment.

Wayne Velicer, my major professor and advisor must be praised as much for his patience as for his clear advice and direction. He was saddled with a particularly distractable student and suffered in silence, almost.

Finally I must thank my wife, Melanie. Her love has been complete and unfailing, her support continuous and her friendship treasured more than I've ever let her know. Thank you, Mel. 


\section{PREFACE}

Determining the number of principal components to retain has been a troublesome applied research problem. There are many methods to choose from and little evidence of any one rule's superiority. Dr. Velicer's work on a particular rule spurred my interest in the question of examining the accuracy of any such rule. Solving the problem proved more difficult than formulating it. Armed with a FORTRAN manual, episodic drive and hope, the work progressed. The results are more encouraging than had been expected, less clear than had been hoped.

Determining the number of principal components to retain will still be a troubling applied research problem, but hopefully less so. 


\section{TABLE OF CONTENTS}

\section{Page}

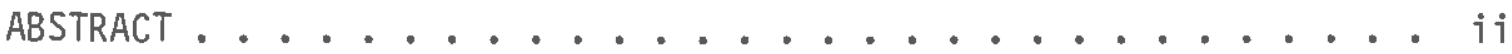

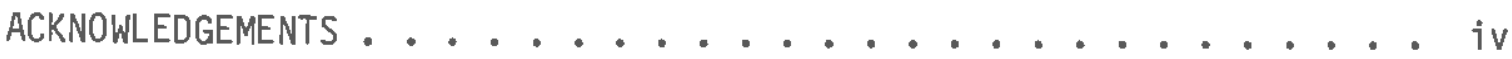

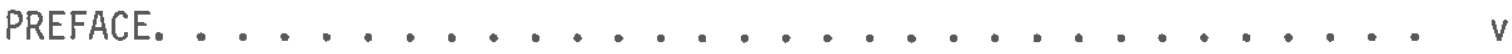
TABLE OF CONTENTS. .............................. vi LIST OF TABLES ............................. vii

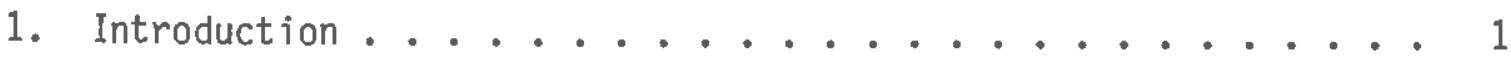

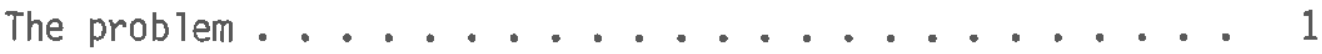

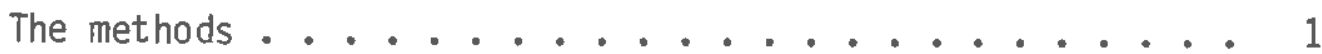

Variables effecting the number of components. . . . . 11

Data generation ................. 13

2. Method .................. 14

Procedure ........................ 14

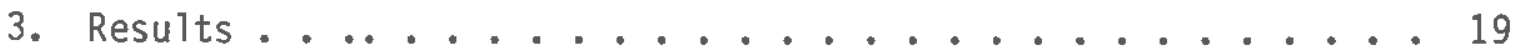

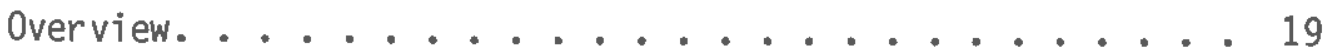

Over and under estimation ............... 23

Percent correct ................... 24

4. Discussion ................... 25

5. References........................ 30 


\section{LIST OF TABLES}

$\underline{\text { Page }}$

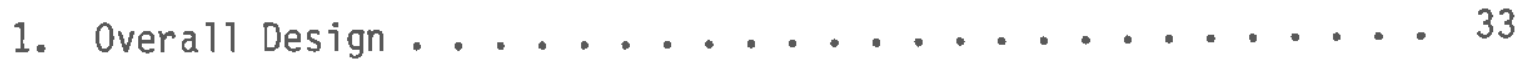

2. Number of Variables per Component. .......... 34

3. Mean Differences at Sample Size $=75$, Component Saturation .50 35

4. Mean Differences at Sample Size $=150$, Component Saturation .50 .36

5. Mean Differences at Sample Size $=450$, Component Saturation .50 .37

6. Mean Differences at Sample Size $=75$, Component Saturation .80 .38

7. Mean Differences at Sample Size $=150$, Component Saturation .80 . 39

8. Mean Differences at Sample Size $=450$, Component Saturation .80 . 40

9. Mean Differences at Levels of Number of Components . . . . . 41

10. Mean Differences at Levels of Sample Size. . . . . . . . . 42 
It is often desirable to represent a large set of variables by some smaller set which still preserves the essential original information. Horst (1965) and Van de Geer (1971) discuss principal component analysis (PCA) as one method of approaching this problem. This study addresses an aspect of this use of PCA. A common problem within principal component analysis is the determination of the number of components to retain (Velicer, 1976; Gorsuch, 1974). This problem is particularly acute when some method of rotation is performed upon the retained components. That is, if an unrotated solution is accepted, the structure of the retained components is not affected by the number of retained components. However, in rotated solutions, the retention of a few more or of a few less components may drastically change the rotated factor structure (Kaiser, 1961). A variety of methods for determining the "appropriate" number of components to retain have been suggested (Kaiser, 1960; Humpreys \& Montane11i, 1975; Catte11, 1966; Joreskog, 1962; Bartlett, 1950, 1951; Velicer, 1976). It has been found the suggested decision methods do not result in the retention of the same number of components (Cattell \& Vogelman, 1977; Linn, 1968).

\section{The Methods}

Given a set of $p$ observed variables how may they be expressed with a smaller set $(m<p)$ of variables such that all the essential information is retained? Two procedures have commonly been employed to resolve this data reduction issue; factor analysis and principal component analysis. 


\section{Factor Analys is}

Traditionally classical factor analysis or common factor analysis (CFA) has been employed to express a set of variables through a smaller set. The factor analytic model specifies a $p \times p$ correlation (or covariance) matrix may be accounted for by $m$ common factors and $P$ unique factors. This model may be expressed as

$$
R=F F^{\prime}+U^{2}
$$

where: $R$ is the $P \times P$ correlation matrix, $F$ is a $p \times m$ matrix of weights for the common factors and $U^{2}$ is the $p \times p$ diagonal matrix of weights for the unique factors. It is important to note $m$ is frequently assumed to be known for the derivation of these procedures.

\section{Principal Component Analys is}

Hotelling (1933) introduced this procedure. The first principal component, $Y_{1}$ is defined as the weighted combination of the $p$ observed $X_{i}$ variables $(i=1 \ldots p)$ which has the greatest sample variance under the constraint the weight vector, $A_{1}$ is of unit length, i.e.,

$$
\begin{array}{ll} 
& Y_{1}=A_{11} x_{1}+\ldots+A_{1 p} x_{p} \\
\text { and } & A_{1} A_{1}=1 .
\end{array}
$$

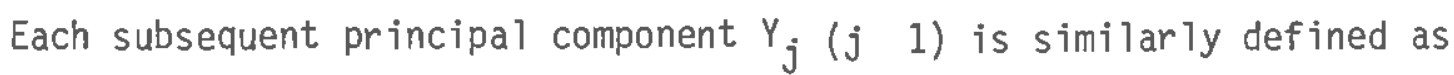
the weighted combination with maximum variance and unit length vector $A_{j}$ such that:

$$
\begin{aligned}
A^{\prime}{ }_{j} A_{j} & =1 \\
\text { and } \operatorname{cov}\left(Y_{j}, Y_{j}\right) & =0 \\
\text { or } \quad A^{\prime}{ }_{j} A_{j} & =0 .
\end{aligned}
$$

Thus each subsequent component has maximum variance and is defined orthogonal to all previous components. 
The principal component solution may be viewed as an eigen decomposition of the $p \times p$ sample variance-covariance matrix (C),

$$
C=L D^{2} L^{\prime}
$$

where the columns of $L$ correspond to the weights (eigen vectors) and where the diagonal values of $D^{2}$ correspond to the total variance of $C$ accounted for by each component (eigen values). Typically the standardized covariance matrix $R$ (the $p \times p$ correlation matrix) is used. On those occasions when a parsimony model is employed some first m (m p) components are retained. For this solution of $m$ principal components, the component pattern may be written as

$$
A=L D
$$

where $D_{c}$ contains the first $m$ eigen roots and $L$ contains the corresponding first $m$ eigen vectors. Kaiser (1970) reported on the widespread use of PCA in this manner. Velicer $(1974,1976,1977)$ has shown this use of PCA and CFA result in essentially equivalent solutions. Given this choice of methods it is important to note an issue has been raised which casts some doubt upon the factor analytic model. The issue revolves around the simultaneous estimation of $\mathrm{F}$ and $\mathrm{U}^{2}$ in equation (1). Guttman (1955) and Schonemann and Wang (1972) discuss this issue of factor indeterminacy. It is concluded indeterminacy is inherent in the CFA procedure and forces a reevaluation of the CFA model. In light of the indeterminacy inherent in the CFA model, the wide usage of the PCA procedure and the comparability of results across the two methods, this proposal will focus upon the PCA procedure.

Within the PCA procedure a number of issues pertaining to its appropriate use arise. Two of the more relevant issues for applied use 
of PCA are:

1) choosing the type of rotation to use on the retained components, and

2) determining the number $(m)$ of components to retain.

Kaiser (1970) reports orthogona varimax rotation is the most common form employed. The choice of the method of rotation, particulariy with regard to the issue of oblique vs. orthogonal seems to be related more to the researcher's preconceptions concerning the content area under investigation than to empirical/theoretical discussions of an optimal rotation method. On the other hand, there appears to be a rational belief that there is an appropriate number $(m)$ of components to retain to best express the set of $p$ variables. Further, the number of components retained affects the structure of any rotated solution. This study focussed on the issue of the number of components to retain.

Methods of Determining the Number of Components to Retain

Gorsuch (1974) categorized decision methods for determining the appropriate number of components into Mathematical, Statisticat, and Non-Triviat Contribution Methods. Methods within each of these categories have something to recommend them and will be examined in turn.

\section{Mathematica T Methods}

I. Perhaps the most popular method, certainty the most common ly employed method, is to retain the components with eigenvalues greater than 1.0. This method was derived from Guttman's (1954) classic work concerning three lower bounds for the number of image components. Kaiser and Caffrey (1965) and Kaiser (1970) have extended the rationale of this method. It should be noted both the common usage of the eigenvaTues 
greater than 1.0 method and some of its intrinsic qualities have troubled investigators. This rule will be referred to below as $\mathrm{K} 1$.

Kaiser (1961) summarized the reason for employing K1 as follows:

1) Algebraic criteria of necessity; following Guttman, Kaiser sees the rule as implicit in his formulations.

2) Psychometric criteria of reliability; "...for a principal component to have positive Kuder-Richardson reliability it is necessary and sufficient that the associated eigenvalue be greater than one...".

(3) Psychological criteria of meaningfulness; Kaiser states "... I have found...the number of eigenvalues greater than one...corresponding almost invariably, in a great number of studies, to the number of factors which practicing psychologists were able to interpret."

Gorsuch (1974) notes many users employ the K1 rule to determine the number of components rather than as a lower bound as originally presented. Difficulties associated with this use are noted by Mote (1970) and Humpreys (1964) who argued that rotation of a greater number of components resulted in more meaningful solutions. They imply the relatively blind use of the $\mathrm{K} 1$ rule therefore, may sometimes lead to the retention of too few components.

Linn (1968) and others (Catte11 \& Jaspers, 1967; Browne, 1968), however, have found the number of components retained by this method often also overestimates the known underlying component structure. Gorsuch (1974) reports the number of components retained by $K 1$ is commonly between one third and one fifth the number of variables included in the correlation matrix. This functional relationship of retained components to the number of variables is detrimental to the accurate estimation of the underlying component structure. The KI method, therefore, although commoniy used, is believed by some critics to sometimes underestimate and by others to sometimes grossly overestimate the number of components, the latter particularly when 
there are a large (e.g., P 50) number of variables involved.

II A second mathematical method is suggested by Velicer (1976). The matrix of the partial correlations of the variables is computed. The average of the squared partial correlation is calculated after each of the $m$ components is partialed out. The minimum average of the squared partial correlation indicates the stopping point for this method. That is, when the average squared partial correlation reaches a minimum, the number of components partialed out is the number of components to be retained. In explanation of why the value of the average of the squared partial correlations, after each component is partialed out, decreases and then increases, Velicer (1976) points out, "consider the general form of a partial correlation equation:

$$
r_{i j} \cdot y=\frac{r_{i j}-r_{i y} \cdot r_{i j}}{\left(\left(1-r_{i}^{2} y\right)\left(1-r_{j}^{2} y\right)\right)^{1 / 2}}
$$

where $i, j$ represent any two of the $p$ observed variables and $y$ represents a component. With no loss of generality, we assume $r_{i j}, r_{i y}$ and $r_{j y}$ to be positive. The value of the partial correlation will decrease as long as the numerator decreases faster than the denominator. The value of the correlation will increase whenever the reverse is true; for example, when $r_{i y}$ is large and $r_{j y}$ is small. Such a situation would occur if a component has a high correlation with only one variable and near zero correlations with the others.." . Thus the nature of the partial correlation equation dictates that the averaged squared partials will continue to decrease until a unique factor is partialed out. At that point, the averaged squared partial will increase. Therefore, the $m$ retained components will contain no unique 
components. This method, to be referred to as the Minimum Average Partial Method (MAP), is congruent with the factor analytic concept of "common" factors. Velicer (1976) points out the method is exact, can be applied with any covariance matrix and is logically related to the concept of factors as representing more than one variable. It is expected MAP will often produce fewer components than will the K1 method, particularly when the number of variables is large. A relatively recently introduced method, the average-squaredpartials method has not been examined systematically to date.

\section{Statistical Methods}

I. Bartlett $(1950,1951)$ suggested a statistical test of the null hypothesis that the remaining eigenvalues are equal. Starting with the first component each is excluded from the test in turn until the null hypothesis fails to be rejected. The first m excluded components prior to the retention of the nu11 hypothesis are the retained components. 
Bartlett presents the formula:

$$
V=-K \ln B
$$

where: $\quad K=N-(2 n+5) / 6-(2 m / 3)$

and

$(m+1)(m+2) \cdots(P)$

$B=$

$(n-m)$

$$
\left[\frac{((m+1)+(m+2)++n)}{n-m}\right]
$$

while: $\quad \mathrm{N}=$ the sample size

$P=$ the number of variables and

$m=$ the number of components already retained

In = natural loganthm.

The statistic $V$ is distributed as chi2 with degrees of freedom (df) equal to:

$$
(P-m-1)(P-m+2)
$$

2

where: $P=$ the number of variables

$\mathrm{m}=$ the number of components already extracted

$\mathrm{n}=$ the sample size.

Bartlett's method appears sensitive to the number of subjects employed. Gorsuch (1975) argued that as the number increases, the tests of significance become more powerful and, therefore, less and less substantial differences between eigenvalues are found to be significant (Gorsuch, 1975). This can lead to the retention of more components as a 
function of the number of subjects, other things being equal. It must also be recalled, however, as the sample size increases the estimates of equal population eigenvalues will become increasingly accurate. This increased accuracy leads to smaller differences between the estimated eigenvalues. It may be the case, with reasonable ranges of sample size, this increased accuracy offsets the increased power of the Bartlett test when the population eigenvalues are actually equal.

\section{Non-Trivial Contribution Methods}

I. The percent of total variance accounted for by the extracted components is perhaps the oldest guideline for determining the number of components to retain. Investigators commonly compute the cumulative percent of variance accounted for after each component is extracted. Components are retained when the process has accounted for 75,85 , or some other arbitrary percent of the total variance. Typically, extraction is stopped when the arbitrarily large proportion is accounted for and when extraction of the next component would contribute little additional variance. However, the arbitrary setting of a goal percentage has neither a firm logical nor a firm mathematical basis. It is usefui to note if $p$ correlated variables are augmented by a set of uncorrelated variables the proportion of variance accounted for drops while the meaningfulness of the relationships remains the same. The presence of uncorrelated variables, therefore, would directly affect the number of retained components. 
II Even simpler than examining the percent of variance accounted for is an examination of the eigenvalues themselves. Cattell (1966) discusses this procedure which he called the "scree" test. "A basic rationale for the scree test is that the battery of variables is measuring a limited number of factors well and a larger number of trivial, specific and error factors much less well.... The predominant factors account for most of the variance and are large, whereas the other factors are quite numerous but sma11.... The substantive factors will be extracted first and the smaller trivial factors will be removed later". (Gorsuch, 1974). The test is simple to apply. The eigenvalues are plotted and those falling above a straight line fit through the smaller values are retained. Complications which can occur include:

1) Gradual slope from lower to higher eigenvalues with no break point in the line.

2) More than one break point in the line.

3) More than one suitable line drawn through the low values. The scree test is most effective when strong components are present with little confounding due to error or unique factors. Tucker, Koopman and Linn (1969) found the scree test to be correct in 12 of 18 cases. Cliff (1970) found it to be accurate, particularly if questionable components are included (Gorsuch, 1974). Cattell and Jaspers (1967) found the test to be correct in 6 of 8 cases. Further, Cliff and Hamburger (1967) found more definite breaks with larger ( $N=$ 400 vs. $N=100$ ) sample sizes and Linn (1968) concurred in this conclusion. Use of the scree test always involves issues of interrater reliability. Cattell and Vogelmann (1977) have shown interrater 
reliability to be good among naive and among expert judges. They have also shown the test to be accurate over 15 systematically differing analyses. Horn and Engstrom (1979) have noted the underlying similarity of Bartlett's $X^{2}$ test and the scree method. Both tests are based on an analysis (one statistical, the other visual) of the essential equality of the "remaining" eigenvalues.

Variables Affecting the Number of Components

This study's examination of the effectiveness of various decision methods will include both Mathematical Methods. The K1 method was included because of its widespread use and the MAP method because of its unambiguous solution and its relation to "common factor" concepts. Bartlett's statistical method was included in this study because it is the only statistical method appropriate for PCA solutions. The scree test was included as the non-trivial contribution method because of its apparent simplicity and its reported validity. Each of these methods may be differentially affected by several different variables including sample size, the number of variables, the degree of component identification and its saturation. The robustness of the four rules in question across these variables is a central focus of this study and may prove to be a useful criteria in choosing among the methods.

\section{Sample size}

Depending upon the decision method employed, it is clear the number of subjects may affect the accuracy of the decision to retain some number of components. Sample size is determined by both the practical applied considerations and the need for accurate estimation. The sample size must be large enough to allow an adequate estimation of the 
relationship between the variables. On the other hand, in applied settings large samples may be too expensive to be practical.

Number of variables

With the development of computer technology and software, larger and larger correlation matrices have been submitted to PCA. PCA's of personality inventories at the item level, for instance, often involve 80 to 100 variables. Analys is of 200 variable sets is becoming common.

\section{Component saturation}

Linn (1968) has demonstrated the underlying component saturation affects decision methods. Underlying components made up exclusively of high loading (e.g., .80) resulted in more retained components, by various decision methods, than did components exclusively made up of low loadings (e.g., .40).

\section{Component identification}

The accurate identification of a component may depend upon the number of variables which load on that component. Components identified by less than three variables are often of limited interest. Further, rules may not be capable of consistently retaining components identified by so few variables. The number of variables loading upon each component could differ from component to component. The impact of this affect is unclear for any of the rules under consideration. $n$ each of the decision methods. 
Method of Data Generation

Studies of the effectiveness of the various decision methods may be categorized into one of two types. The more common type of study employs real data representing either new work or "classic" studies. These studies employ some logical criteria concerning the appropriate number of components and compare the performance of the proposed decision method to the logically arrived at value (e.g., Velicer, 1976; Humphreys \& Montane17i, 1975). These studies, in employing an arbitrary logical criterion, may inaccurately estimate the performance of the decision method in question. The second type of study employs correlation matrices generated from component structures entirely under the control of the investigator (e.g., Tucker, Koopman \& Linn, 1969; Catte11 \& Vogelmann, 1977). These studies have the advantage of a known criterion against which to measure the performance of the decision method. They are, however, open to the criticism that their generated matrices, although conforming to a mathematical model may not well represent real data and thus lead to inappropriate conclusions (Tucker, Koopman \& Linn, 1969).

The question of a rule's accuracy can not be examined without a known criterion. Although logical arguments can be mounted to defend the number of components present in some data sets, these arguments are always open to question. For the assessment of the impact of various conditions upon a rule's accuracy, generated data of a known number of components is preferable. The issue of generalization to real data sets is an important but separate issue. It may be independently addressed in the particular way the data is generated. 
Method

\section{Procedure}

As indicated above, the number of variables employed, the sample size, the number of components and the component saturation may effect the results of the various decision rules. This study examined the effect of three levels of sample size, three levels of number of variables, three levels of numbers of components, and two levels of component loadings on each of four decision methods (K1, MAP; BART; SCREE).

The levels within each factor were chosen to represent applied research conditions. Small sample sizes are often a necessity. Large samples lead to much greater accuracy of estimation. However, the impact of increasing the sample size decreases as the sample becomes Targer and larger.

The range of 75 to 450 was chosen to represent small and moderately large samples. An intermediate value of 150 was also included.

Data reduction techniques are typically not required on small data sets. The use of PCA in test construction, on the other hand, often involves 100 or more variables. Data sets of 150 or more variables are no longer rare. To represent this range, 36 variables were chosen as the smallest data set, 72 variables as a moderate set and 144 variables as the largest set. This range appears representative of applied use and wide enough to impact any rule sensitive to the number of variables.

Two components are required for rotation and three or more are often assumed present. Few theoretical constructs call for more than 15 or 20 orthogonal components. Given the range of variables indicated above and the desire to have an equal number of variables identifying 
each component, 3,6 and 12 were chosen as the levels of the number of components.

Loading below .30 or .35 are typically ignored in applied uses of PCA. Loadings of over .85 are rarely found. The lower level of component saturation was set at .50 to avoid trivial loadings while still representing variables which shared only a moderate proportion of their variance with a component. The upper level was set at .80 to represent high loading variables. Linn (1969) has found a similar range (.40 to .80$)$ broad enough to differentially effect decision rules.

Ten correlation matrices were generated for each combination of the $3 \times 3 \times 3 \times 2$ factors outlined above. No matrices were generated for those cells where the number of variables was 144 and the sample size was 75 . Each population correlation matrix was determined as follows: One appropriate population component matrix was created in accordance with the level of the "number of variables factor", the level of the "saturation factor" and of the "number of components factor" under consideration. Pre-multiplying by its transpose (CC') resulted in a matrix $R^{*}\left(C C^{\prime}=R^{*}\right)$. Substitution of ones into the diagonal of $R^{*}$ introduced error and produced a population correlation matrix $R\left(R^{*}+\right.$ $\left.D^{2}=R\right)$. The introduction of ones in the diagonal of $R$ raised it to full rank thereby allowing subsequent analysis. Sample correlation matrices based upon this population correlation matrix and upon the level of the "number of observations factor" (sample size) were generated through a computer program (Montanelli, 1975). Ten sample correlation matrices were generated for each cell. For example, in an eight variable $(p=8)$, two component $(m=2)$ case with high component 
saturation, the underlying component structure $C$ would be:

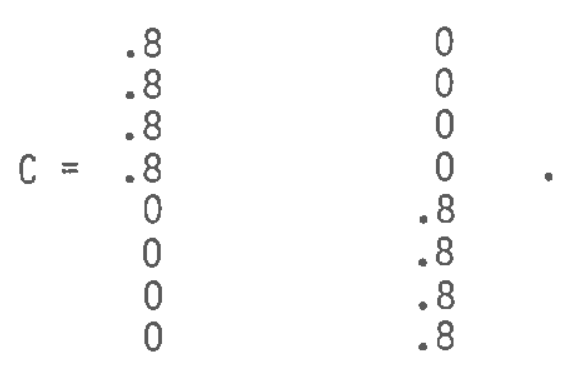

Given this component structure,

$$
R^{\star}=C C^{\prime}=\begin{array}{rrrrrrrr}
.64 & .64 & .64 & .64 & 0 & 0 & 0 & 0 \\
.64 & .64 & .64 & .64 & 0 & 0 & 0 & 0 \\
.64 & .64 & .64 & .64 & 0 & 0 & 0 & 0 \\
.64 & .64 & .64 & .64 & 0 & 0 & 0 & 0 \\
0 & 0 & 0 & 0 & .64 & .64 & .64 & .64 \\
0 & 0 & 0 & 0 & .64 & .64 & .64 & .64 \\
0 & 0 & 0 & 0 & .64 & .64 & .64 & .64 \\
0 & 0 & 0 & 0 & .64 & .64 & .64 & .64
\end{array}
$$

and

$$
\text { D2 }=\begin{array}{cccccccc}
.36 & 0.0 & 0.0 & 0.0 & 0.0 & 0.0 & 0.0 & 0.0 \\
0.0 & .36 & 0.0 & 0.0 & 0.0 & 0.0 & 0.0 & 0.0 \\
0.0 & 0.0 & .36 & 0.0 & 0.0 & 0.0 & 0.0 & 0.0 \\
0.0 & 0.0 & 0.0 & .36 & 0.0 & 0.0 & 0.0 & 0.0 \\
0.0 & 0.0 & 0.0 & 0.0 & .36 & 0.0 & 0.0 & 0.0 \\
0.0 & 0.0 & 0.0 & 0.0 & 0.0 & .36 & 0.0 & 0.0 \\
0.0 & 0.0 & 0.0 & 0.0 & 0.0 & 0.0 & .36 & 0.0 \\
0.0 & 0.0 & 0.0 & 0.0 & 0.0 & 0.0 & 0.0 & .36
\end{array}
$$


so that the resulting population correlation matrix $\mathrm{R}$ would be

$\mathrm{R}=\begin{array}{rrrrrrrr}1.0 & .64 & .64 & .64 & 0 & 0 & 0 & 0 \\ .64 & 1.0 & .64 & .64 & 0 & 0 & 0 & 0 \\ .64 & .64 & 1.0 & .64 & 0 & 0 & 0 & 0 \\ .64 & .64 & .64 & 1.0 & 0 & 0 & 0 & 0 \\ 0 & 0 & 0 & 0 & 1.0 & .64 & .64 & .64 \\ 0 & 0 & 0 & 0 & .64 & 1.0 & .64 & .64 \\ 0 & 0 & 0 & 0 & .64 & .64 & 1.0 & .64 \\ 0 & 0 & 0 & 0 & .64 & .64 & .64 & 1.0\end{array}$

Montanelli's (1975) program was then employed to generate ten sample correlation matrices from this population matrix.

It is important to note all rules were $100 \%$ accurate in identifying the number of components in the population matrices (MAP, KI and SCREE through application, BART by definition). This convergence on the population parameter reaffirms its appropriateness as a criterion when examining the sample results.

Principal component analysis was then performed on each of the resulting 480 sample correlation matrices. At the time this analys is was performed, the number of components to be retained was determined using each of the three calculable rules (K1, MAP and BART). The Bartlett's test was performed at an alpha level of .05 in all cases.

Plots of the eigenvalues for each analysis were obtained. These plots were examined by raters trained in the scree method. The two raters were graduate students in psychology. They were briefly trained also in the scree procedure but uninformed as to its purpose. The raters were also naive as to the purpose of the experiment and had no prior applied experience with the scree test. The graphs were presented to each rater independently and they were unaware of each 
other's responses. The graphs themselves were computer generated on 8 $1 / 2^{\prime \prime} \times 14^{\prime \prime}$ sheets of paper and contained no indication of what they represented. The raters achieved high inter-rater reliabiltiyl = .94) across the 480 samples examined. An experienced expert judge, uninformed as to the purpose of the experiment but familiar with the use of the scree test, rated one sample from each of the 48 cells. The correlation between the judge's ratings and the mean rating of the raters was $r=.86$.

Table 1 presents an outline of the overall design.

Insert Table 1 here

Different numbers of variables load on each component across the structures examined. Table 2 summarizes this result.

Insert Table 2 here

This confounding of number of variables, components and variables per component is unavoidable. Any other specification coupled with the absence of unique variables would lead to similar but perhaps less obvious confounding. 


\section{Results}

\section{Overview}

The mean number $(X)$ of components retained by each rule in each cell of the overall design was computed. This mean was then subtracted from the population criterion. Positive mean difference (d) scores, therefore, indicate underestimation of the population value while negative mean difference scores indicate overestimation. Table 3 presents a summary of these results for those cells in which the component saturation was equal to .50 and the sample size was equal to 75. As Table 4 through 8 follow the same format as Table 3, a detailed description will only be given for Table 3 .

Insert Table 3 here

The first row of Table 3 examines the performance of the four decision rules (MAP, K1, BART SCREE) under the condition of three population components $(m=3)$ and 36 variables $(p=36)$ in the correlation matrix. Under this condition MAP retained an average of 3.0 component and, thus, had a mean difference of 0.0 from the criterion. K1 retained 12 components for a difference score of -9.0 , an overestimation. BART retained an average of 2.0 components for a mean difference of 1.0, an underestimation. SCREE retained an average of 3.1 components for a mean difference of -.1 , an overestimation. Row 2 represents the same values for each decision rule when the three 
components were based on 72 variables $(p=72)$. Under these conditions, MAP overestimated slightly $(d=-.5), \mathrm{K} 1$ and BART overestimated greatly ( -20.8 and -22.2 respectively) and SCREE overestimated slightly $(d=-.1)$. Row 3 presents a summary of the rules' performance across 36 and 72 variables when the number of components was three $(m=3)$, the sample size was 75 and the component saturation was .50 . From this row it can be seen MAP and SCREE overestimated slightly ( $d=-.25$ and -.1 respectively) while $K 1$ and BART have overestimated the number of components to a much larger degree ( $d=-14.90$ and -11.0 respectively). A similar examination of rows 4 through 6 indictes, when the number of components is $\operatorname{six}(m=6)$ and the number of variables is $36(p=36)$, MAP and BART somewhat underestimated the number of components $(d=1.70,2.0)$, SCREE slightly overestimates and $\mathrm{K} 1$ greatly overestimates the number of components ( $d$ $=-.45$ and -7.30 respectively). Across both levels of the number of variables factor $(p=36,72)$ MAP slightly underestimated $(d=.90)$, SCREE slightly overestimated $(d=-.42)$ while K1 and BART greatly overestimated ( $d=-13.0$ and -15.30 respectively) the criterion value. A similar examination of rows 7 through 9 indicates, across both levels of the number of variables factor, MAP greatly underestimated ( $d=$ 9.0), SCREE moderately underestimated $(d=4.15)$ while $K 1$ and BART greatly overestimated ( $d=-8.02$ and -7.15 respectively) the population value. Row 10 presents the mean difference from the population value across all levels of the number of components and number of variables factors when the sample size was equal to 75 and the component saturation was .50 . Row 10 indicates MAP and SCREE underestimated somewhat $(d=3.22$ and 1.21 respectively) while K1 and BART overestimated greatly $(d=-11.98$ and -11.15$)$. It is 
important to note much of the underestimation by MAP and SCREE occurred in those cells where the population value for number of components was 12. Overestimation by BART occurred only when the number of variables was 72. This overestimation was great enough to overwhe $1 \mathrm{~m}$ the more moderate performance of BART under other conditions. K1, however, consistentiy overestimated and the amount of overestimation appears to have been related to the number of variables. The more variables involved, the more components retained by $\mathrm{K} 1$. It should be noted no $\mathrm{p}$ $=144$ matrices were examined in Table 3 due to the small sample.

Table 4 is presented in the same way as Table 3 . The sample size has been increased to 150 , however, so $p=144$ matrices have been included. Under these conditions the performance of MAP, BART and SCREE improved but K1 continued to overestimate greatly. Across the

three levels of number of components and number of variables (row 13) SCREE slightly underestimated $(d=.54)$, MAP and BART moderately underestimated $(d=1.61$ and 1.21$)$ while $K 1$ greatly overestimated $(d=$ -21.08). It is important to note that much of the underestimation by MAP, BART and SCREE occurred when the population criterion was 12 and the number of variables was 36 (row 9). K1's overestimation appeared again to be closely linked to the number of variables. For each increase in the number of variables at any one level of the number of components there appeared to be a related increase in the number of components retained by $\mathrm{K} I$ in Table 4 . 
Table 5 is presented in the same way as Tables 3 and 4 . In this

Insert Table 5 here

case the sample size is 450 . BART and SCREE performed well across the three levels of numbers of components $(3,6,12)$ and numbers of variables $(36,72,144)$. Both BART and SCREE underestimated by a minute amount $(d=.01$ for both) (Row $=13)$. MAP performed perfectly except in the case of 12 population components and 36 variables. Its underestimation in this cell resulted in a small overall underestimation of 1.22. K1 consistently overestimated the population criterion $(d=-18.0)$. The overestimation appeared again to be related to the number of variables.

Tables 6,7 and 8 parallel Tables 3,4 and 5 with an increase in component saturation from .50 to .80 . Table 6 indicates BART again greatly overestimated when the number of variables is equal to 72 and

\section{Insert Table 6 here}

the sample size was 75 (rows $=2,6,10$ ). K1 continued to overestimate the population criterion but to a much lesser degree. MAP and SCREE very closely approximated the population criterion in all cells.

Table 7 summarizes the results when the sample size was increased 
to 150 . There was some underestimation by BART when the number of

\section{Insert Table 7}

variables was 144. SCREE and MAP performed very well with overall overestimation of only -.01 and -.08 respectively. $\mathrm{K} 1$ continued to overestimate but this error was essentially restricted to the case of $p$ $=144$.

Table 8 represents what theoretically should have been a "best case" for all the rules employed. The component saturation was high (.80) and the sample size was reasonably large $(N=450)$. MAP performed perfectly with no mean over or under estimations, for an overall d of 0.0 . SCREE closely followed with a few small mean over

Insert Table 8 here

and underestimation for an overall d of 0.01 . BART, while still slightly underestimating when $p=144$, had an overall underestimation of only 0.03 . Kl continued to overestimate but only in the case of $p=$ 144 when the number of components was equal to 12 . K1 had an overall overestimation of.- .22 across Table 8.

Over- and Underestimation

Table 9 presents a summary of the overestimation and underestimation 
performance of each decision rule at each level of component saturation. At .50 saturation MAP, BART, and SCREE appear more likely

Insert Table 9 here

to underestimate the number of components then to overestimate. At .80 saturation the reverse appears true but to a much smaller extent. KI consistently overestimated though to a lesser degree at the higher level of saturation.

\section{Percent Correct}

Table 10 provides the percent correct identification of the population criterion value by each rule at each level of component saturation. It appears MAP and SCREE matched the criterion value with about equal, relatively high frequency. K1 and BART performed similarly

to each other at .80 saturation. BART performed only marginally at .50 saturation while KI virtually never correctly identified the population criterion value at that saturation level. 


\section{Discussion}

This study attempted to examine the performance of four decision rules for determining the number of principal components to retain. Employing known population correlation matrices, ten samples were drawn for each of 48 cases. These 48 cases represented four systematically varying parameters. The four parameters were the number of components $(m=3,6,12)$, the number of variables $(p=36,72,144)$, the sample size $(N=75,150,450)$ and the component saturation $(C S=.50, .80)$. The four decision rules employed were KI, SCREE, MAP and Bartlett's test. All four rules retained the appropriate number of components when applied to the population correlation matrix.

The study employed very simple population correlation matrices. That is, no unique or complex variables were included. These matrices all involved only large common components and error. This situation conformed exactly to the assumptions of all four rules. This antiseptic condition was seen as a good first comparative testing ground for these decision rules. Weak performance by any decision rule under these conditions would appear to be strong evidence against the continued applied use of that rule.

The $\mathrm{KI}$ rule was found to have consistently overestimated the number of components. This finding parallels those of Linn (1968), Cattell and Jasper (1967) and Browne (1968). At low saturation, the number retained often fell in the $1 / 3$ to $1 / 5 \mathrm{p}$ range discussed by Gorsuch (1974). As the number of variables increased so did the number of components retained. Particularly at .50 loadings, the number of components retained appeared more related to the number 
of variables than to the number of components in the structure. This finding is clearly contrary to Mote's (1970) and Humphreys' (1964) expectation that the $\mathrm{K} 1$ rule would retain too few components. Given the apparent functional relationship of the number of components retained to the number of variables, it is difficult to recommend the continued use of the $\mathrm{K} 1$ rule.

The BART rule performed well in most cases except when the number of variables closely approached the sample size. It had been expected BART would overestimate the number of components at large sample sizes (Gorsuch, 1974). This did not occur. The range of sample size in this study was chosen to reflect applied situations. It is possible, at greatly increased sample sizes, BART would begin to systematically overestimate. The difficulty of choosing an "appropriate" significance level in practice is an applied limitation of the BART rule (Horn \& Engstrom, 1979) which was not addressed in this study.

Bartlett's test was most inaccurate at the smallest sample sizes when the number of variables approached that sample size (i.e., $p=72$, $N=75$ ). A maximum likelihood test (Joreskog, 1962) similar to the Bartlett test involves a specific correction factor in situations like this. No such correction exists for Bartlett's test. Until such a correction is developed the Bartlett test should not be employed in such situations.

As the first comparative test of the MAP rule, this study demonstrated the procedures's general accuracy. MAP consistently underestimated the number of components in one situation. This occurred when the number of components was large relative to the number of variables and the component saturation was low (i.e., $m=12, p=36$ and 72 and $C S=.50)$. This may have occurred because the common 
component in such a case is less we 11 defined than the unique part of a single variable. An investigator who wished to retain such components would be advised to employ an alternate procedure. This study indicates MAP is a viable decision rule worthy of future consideration. Its unequivical stopping point and its relation to "common factors" argue for further examination of the rule.

The SCREE test generally performed best across all settings. These results expand on those of Cattell and Vogelman (1977) concerning the SCREE's accuracy. High interrater reliability was attained. The accuracy of this rule relative to the three others across the 48 situations examined is perhaps the strongest support available for the more widespread use of the SCREE procedure. Certainly, future research in this area must now include this test.

It appeared, from the 480 observations, component saturation had the greatest impact upon the accuracy of three of the decision rules. Each rule, except BART, performed better at the higher $(.80)$ level of saturation than at the lower $(.50)$ level. It should be recalled components made up of .50 loadings are typically not considered weakly defined. Increases in sample size generally improved rule performance. Increases in the number of variables examined had a dramatic detrimental effect upon K1 but did not appear to negatively effect any other rule. Such increases, in fact, appeared to aid MAP and SCREE. All rules had difficulty identifying 12 components under conditions of small sample size, .50 saturation and 36 or 72 variables. This effect may have been caused by the smaller number of variables identifying these components.

Quite apart from the important impact of these variables upon each of the decision rules, it is striking to note the wide range in 
performance between these rules. SCREE and MAP consistently provided closer estimates of the population criterion value than did BART and K1. The large, persistent overestimation by K1 paired with its sensitivity to the number of variables examined argues strongly against its widespread indiscriminant use. BART's performance was considerably better than K1's, particularly at .50 saturation and at sample sizes of 150 and 450.

Further examination of these rules, particularly SCREE and MAP, under more complex conditions is called for. For instance, applied data sets often include complex variables which load above .50 on more than one component. The impact of such complex variables upon decision rules has not been systematicly examined to date. Second, it is not uncommon to find a single variable loading substantially on a component identified by no other high loadings. These unique variables may lead to a component being retained by some rules but not by others. Third it is also common to find a different number of variables identifying various components within any data set. This inequality may differentially effect decision rules. For instance, in a $p=40$ case two sets of 11 variables could identify each of the first two components and three sets of six variables identify the next three components. Such a multiple break structure may pose problems for rules such as SCREE and BART which search smaller eigenvalues.

Factors such as the three described above may drastically influence the performance of these rules. An examination of at least these factors is needed before a final decision can be reached concerning which of these rules is most appropriate.

Based upon the results of this study, which included samples drawn from simple, well defined population correlation matrices, we can 
conclude there is no evidence supporting the continued use of the K1 rule. The BART procedure was found to generally perform quite accurately except when the number of variables was close to the sample size. In the latter situations BART greatly overestimated the number of components and therefore should not be employed under such conditions. A correction such as that employed by Joreskog (1962) in a maximum likelihood application might remedy this problem. The MAP procedure was generally accurate except when the component saturation was low and few variables defined a component. If an investigator wishes to retain components of that type other procedures may be more appropriate. The SCREE was generally the most accurate rule across all cases. 


\section{$\underline{\text { References }}$}

Bartlett, M.S. Tests of significance in factor analysis. The British Journal of Psychology, 1950, $\underline{3}, 77-85$.

Bart lett, M.S. A further note on tests of significance in factor analysis. The British Journal of Psychology, 1951, 4, 1-2.

Browne, M.W. A note on lower bounds for the number of common factors. Psychometrika, 1968, 33, 2, 233.

Catte11, R.B. The scree test for the number of factors. Multivariate Behavioral Research, 1966, I, 245-276.

Catte11, R.B. \& Jaspers, J. A general plasmode for factor analytic exercises and research. Multivariate Behavioral Research Monographs, 1967, 1-212.

Catte11, R.B. \& Voge1man, S. A comprehensive trial of the scree and KG criteria for determining the number of factors. Multivariate Behavioral Research, 1977, 12, 289-325.

Cliff, N. The relation between sample and population characteristic vectors. Psychometrika, 1970, 35, 2, 163.

Cliff, N. \& Pennell, R. The influence of communality, factor strength and loading size on the sample characteristics of factor loadings. Psychometrika, 1967, 32, 3, 309.

Gorsuch, R.L. Factor Analysis. Philadelphia: Saunders, 1974.

Guttman, L. Some necessary conditions for common factor analysis. Psychometrika, 1954, 19, 149-162.

Harris, M.L. \& Harris, C.W. A factor analytic interpretation strategy. Educational and Psychological Measurement, 1971, 31, 3, 589.

Horst, P. Factor Analysis of Data Matrices. New York: Holt, Rinehart and Winston, 1965.

Hotelling, H. Analysis of a complex of statistical variables into principal components. Journal of Educational Psychology, 1933, 24, 417-441, 498-520.

Humphreys, L.G. Number of cases and number of factors: An example where $\mathrm{N}$ is very large. Educational and Psychological Measurement, $1964, \underline{24}, 457$.

Humphreys, L.G. \& Montanelli, R.G. An investigation of the parallel analys is criterion for determining the number of common factors. Multivariate Behavioral Research, 1975, 193-205. 
Kaiser, H.F. The application of electronic computers to factor analys is. Educational and Psychological Measurement, 1960, 20, $141-151$.

Kaiser, H.F. A note on Guttman's lower bound for the number of common factors. British Journal of Statistical Psychology, 1961, 14, 1-2.

Kaiser, H.F. A second-generation little jiffy. Psychometrika, 1970, 35, 401-415.

Kaiser, H.F. \& Caffrey, J. Alpha factor analysis. Psychometrika, 1965, $30,1-44$.

Law]ey, D.N. The estimation of factor loadings by the method of maximum likelihood. Proceedings of the Royal Society of Edinburgh, 1940, $60,64-82$.

Linn, R.L. A Monte Carlo approach to the number of factors problem. Psychometrika, 1968, 33, 37-71.

Montanelli, R.G., Jr. A computer program to generate sample correlation and covariance matrices. Educational and Psychological Measurement, 1975, 35, 195-197.

Mote, T.A. An artifact of the rotation of too few factors: Study orientation vs. trait anxiety. Revista Interamericana de Psicologia 4(3-4): $171,1970$.

Schonemann, P.H. \& Wang, M. Some new results on factor indeterminacy. Psychometrika, 1972, 37, 61-91.

Tucker, L.B., Koopman, R.F. \& Linn, R.L. Evaluation of factor analytic research procedures by means of simulated correlation matrices. Psychometrika, 1969, 34, 421.

Van de Geer, J.P. Introduction to Multivariate Analys is for the Social Sciences. San Francisco: Freeman, 1971.

Velicer, W.F. A comparison of the stability of factor analysis, principal component analys is and rescaled image analysis. Educational and Psychological Measurement, 1974, 34, 563-572.

Velicer, W.F. Determining the number of components from the matrix of partial correlations. Psychometrika, 1976, 41, 3, 321-327.

Velicer, W.F. An emperical comparison of the similarity of principal component, image, and factor patterns. Multivariate Behavioral Research, 1977, 12, 3-22. 
Table 1

Overall Design

Component

Saturation

Number of
Components

75
Sample Size

75

450
150

450

*

*

$\star$

$\begin{array}{lll}75 & 150 & 450\end{array}$

3

36

6

12

3

.50

72

6

12

3

144

6

12

\section{.}

2

3

2

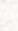

3

36

6

12

3

.80

72

6

12

3

6

12

6

2

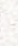

144

12

* No cases were generated for these cells. 


\section{Table 2}

The number of variables per component for the six structures selected.

Total Variables

\begin{tabular}{lll}
36 & $72 \quad 144$ \\
\hline
\end{tabular}

3

$\begin{array}{lll}12 & 24 & 48\end{array}$

Number of

6

$\begin{array}{lll}6 & 12 & 24\end{array}$

Components

$\begin{array}{lll}3 & 6 & 12\end{array}$


Table 3

Mean number of components retained and mean difference from population value for each level of number of variables (p) and components $(m)$. Sample size $=75$. Component saturation $=.50$.

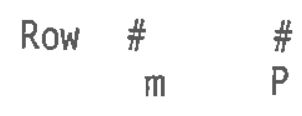

1336

2

3 Sub. Tot.

$4 \quad 6 \quad 36$

5

6 Sub. Tot.

$\begin{array}{lll}7 & 12 & 36\end{array}$

8

72

9 Sub. Tot.

10 Tot. d
MAP

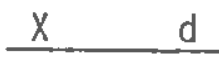

$3.0 \quad 0.0$

$3.5-0.50$

$3.25-0.25$

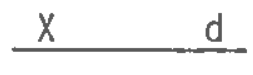

$12.0 \quad-9.0$

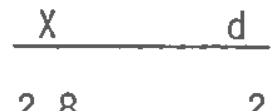

BART

SCREE

$23.80 \quad-20.80$

25.2

$-22.2$

$\begin{array}{lll}.2 & 3.1 & -0.1\end{array}$

$\begin{array}{ll}17.90 & -14.90\end{array}$

14.0

$-11.0$

$3.1-0.1$

$4.3 \quad 1.70$

$13.30 \quad-7.30$

4.0

2.0

$\begin{array}{ll}6.45 & -0.45\end{array}$

$5.9 \quad 0.10$

$24.70 \quad-18.70$

38.60

$\begin{array}{lll}-32.60 & 6.40 & -0.40\end{array}$

5.1

0.90

$19.0-13.0$

21.30

$\begin{array}{lll}-15.30 & 6.42 & -0.42\end{array}$

$1.5 \quad 10.50$

$14.70 \quad-2.70$

3.30

$\begin{array}{lll}8.70 & 7.20 & 4.80\end{array}$

$4.5 \quad 7.50$

$25.80 \quad-13.80$

35.0

$-23.00 \quad 8.50$

3.50

$3.0 \quad 9.0$

$20.02 \quad-8.02$

19.15

$\begin{array}{ll}-7.15 & 7.85\end{array}$

4.15

--. 3.22

$---\quad-11.98$

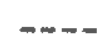

$-11.15$

1.21 
Table 4

Mean number of components retained and mean difference from population value for each level of number of variables $(p)$ and components $(m)$. Sample size $=150$. Component saturation $=.50$.

\begin{tabular}{|c|c|c|c|c|c|c|c|c|c|c|}
\hline \multirow[t]{2}{*}{ Row } & \multirow{2}{*}{$\begin{array}{l}\# \\
\mathrm{~m}\end{array}$} & \multirow[t]{2}{*}{ \# } & \multicolumn{2}{|c|}{ MAP } & \multicolumn{2}{|c|}{$\mathrm{K} 1$} & \multicolumn{2}{|c|}{ BART } & \multicolumn{2}{|c|}{ SCREE } \\
\hline & & & $x$ & d & $x$ & d & $x$ & d & $x$ & d \\
\hline 1 & 3 & 36 & 3.0 & 0.0 & 11.1 & -8.1 & 3.0 & 0.0 & 3.0 & 0.0 \\
\hline 2 & & 72 & 3.1 & -.1 & 22.7 & -19.7 & 3.0 & 0.0 & 3.0 & 0.0 \\
\hline 3 & & 144 & 3.0 & 0.0 & 42.8 & -39.8 & 2.7 & 0.3 & 3.0 & 0.0 \\
\hline 4 & Sub. & Tot. & 3.03 & -.03 & 26.47 & -23.47 & 2.9 & 0.1 & 3.0 & 0.0 \\
\hline 5 & 6 & 36 & 5.3 & 0.7 & 12.4 & -6.4 & 5.2 & 0.8 & 6.2 & -0.2 \\
\hline 6 & & 72 & 6.0 & 0.0 & 24.4 & -18.4 & 6.0 & 0.0 & 6.1 & -0.1 \\
\hline 7 & & 144 & 6.2 & -.2 & 49.3 & -43.3 & 5.8 & 0.2 & 6.0 & 0.0 \\
\hline 8 & Sub. & Tot. & 6.05 & -.15 & 28.7 & -16.7 & 5.67 & 0.33 & 6.0 & 0.0 \\
\hline 9 & 12 & 36 & 1.0 & 11.0 & 14.4 & -2.4 & 6.5 & 5.5 & 7.55 & 4.55 \\
\hline 10 & & 72 & 8.9 & 3.1 & 26.2 & -14.2 & 9.3 & 2.7 & 10.85 & 1.15 \\
\hline 11 & & 144 & 12.0 & 0.0 & 49.0 & -37.0 & 10.5 & 1.5 & 12.45 & -0.45 \\
\hline 12 & Sub. & Tot. & 7.3 & 4.7 & 29.87 & -17.87 & 8.8 & 3.2 & 10.28 & 1.72 \\
\hline 13 & Tot. & d & -- & 1.61 & --- & -21.08 & --- & 1.21 & -.-- & 0.54 \\
\hline
\end{tabular}


Table 5

Mean number of components retained and mean difference from population value for each level of number of variables (p) and components (m). Sample size $=450$. Component saturation $=.50$.

$\begin{array}{cc}\text { Row } & \# \\ m & \text { p }\end{array}$

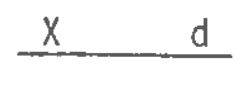

1336

2

3

4 Sub. Tot.

$\begin{array}{lll}5 & 6 & 36\end{array}$

6

72

144

7

8 Sub. Tot.

$\begin{array}{lll}9 & 12 & 36\end{array}$

10

11

72

144

12 Sub. Tot.

13 Tot. d

MAP

$3.0 \quad 0.0$

$3.0 \quad 0.0$

$3.0 \quad 0.0$

$3.0 \quad 0.0$

$6.0 \quad 0.0$

$6.0 \quad 0.0$

$1.0 \quad 11.0$

$12.0 \quad 0.0$

$12.0 \quad 0.0$

$8.33 \quad 3.67$
$9.1-3.1$

$\begin{array}{llll}6.0 & 0.0 & 20.1 & -14.1\end{array}$

$6.0 \quad 0.0$

$13.1-1.1$

$23.2-11.2$

$\begin{array}{ll}45.8 & -33.8\end{array}$

$27.37-15.37$
6.1

6.2

11.8

11.4

12.0

12.0

$0.0 \quad 12.0 \quad 0.0$

$0.2 \quad 12.02-0.02$

SCREE

BART

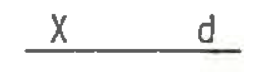

$\begin{array}{lll}-.1 & 3.0 & 0.0\end{array}$

$0.0 \quad 3.0 \quad 0.0$

$\begin{array}{lll}0.0 & 3.0 & 0.0\end{array}$ $-0.03 \quad 3.0 \quad 0.0$

$-.1$

6.0

0.0

$\begin{array}{lll}-.2 & 6.0 & 0.0\end{array}$

$\begin{array}{lll}-1.0 & 6.0 & 0.0\end{array}$

$\begin{array}{llll}6.13 & -.23 & 5.95 & 0.05\end{array}$

$0.6 \quad 12.05 \quad-.05$

$0.0 \quad 12.0 \quad-.0$

$-0.02$

0.01 
Table 6

Mean number of components retained and mean difference from population value for each level of number of variables $(p)$ and components $(m)$. Sample size $=75$. Component saturation $=.80$.

\begin{tabular}{|c|c|c|c|c|c|c|c|c|c|c|}
\hline \multirow[t]{2}{*}{ Row } & \multirow{2}{*}{$\begin{array}{l}\# \\
\text { m }\end{array}$} & \multirow{2}{*}{$\begin{array}{l}\# \\
p\end{array}$} & \multicolumn{2}{|c|}{ MAP } & \multicolumn{2}{|c|}{ K1 } & \multicolumn{2}{|c|}{ BART } & \multicolumn{2}{|c|}{ SCREE } \\
\hline & & & $x$ & d & $x$ & $d$ & $x$ & d & $x$ & d \\
\hline 1 & 3 & 36 & 3.1 & -.1 & 3.1 & -.1 & 3.2 & -.2 & 3.0 & 0.0 \\
\hline 2 & & 72 & 3.1 & -.1 & 6.4 & 3.4 & 39.6 & -36.6 & 3.0 & 0.0 \\
\hline 3 & Sub. & Tot. & 3.1 & -.1 & 5.25 & -2.25 & 21.45 & -18.45 & 3.0 & 0.0 \\
\hline 4 & 6 & 36 & 6.0 & 0.0 & 6.0 & 0.0 & 6.3 & -.3 & 6.0 & 0.0 \\
\hline 5 & & 72 & 6.1 & -.1 & 10.2 & -4.2 & 35.4 & -29.4 & 6.0 & 0.0 \\
\hline 6 & Sub. & Tot. & 6.05 & -.05 & 8.1 & -2.1 & 20.85 & -14.85 & 6.0 & 0.0 \\
\hline 7 & 12 & 36 & 12.0 & 0.0 & 11.9 & 0.1 & 12.1 & -.1 & 12.05 & -.05 \\
\hline 8 & & 72 & 12.2 & -.2 & 13.6 & -1.6 & 34.7 & -22.7 & 12.05 & -.05 \\
\hline 9 & Sub. & Tot. & 12.1 & -.1 & 12.75 & -.75 & 23.4 & -11.4 & 12.05 & -.05 \\
\hline 10 & Tot. & d & -- & .08 & --.- & -1.7 & ---- & -14.9 & --- & -.02 \\
\hline
\end{tabular}


Table 7

Mean number of components retained and mean difference from population vatue for each leve T of number of variables $(p)$ and components $(\mathrm{m})$. Sample size $=150$. Component saturation $=.80$.

\begin{tabular}{|c|c|c|c|c|c|c|c|c|c|c|}
\hline \multirow[t]{2}{*}{ Row } & \multirow{2}{*}{$\begin{array}{l}\# \\
\text { m }\end{array}$} & \multirow[t]{2}{*}{$\#$} & \multicolumn{2}{|c|}{ MAP } & \multicolumn{2}{|c|}{ K1 } & \multicolumn{2}{|c|}{ BART } & \multicolumn{2}{|c|}{ SCREE } \\
\hline & & & $x$ & $d$ & $x$ & d & $x$ & d & $x$ & d \\
\hline 1 & 3 & 36 & 3.0 & 0.0 & 3.0 & 0.0 & 3.0 & 0.0 & 3.0 & 0.0 \\
\hline 2 & & 72 & 3.3 & -.3 & 3.6 & -.6 & 3.0 & 0.0 & 3.0 & 0.0 \\
\hline 3 & & 144 & 3.3 & -.3 & 13.3 & -10.3 & 2.0 & 1.0 & 3.0 & 0.0 \\
\hline 4 & Sub. & Tot. & 3.2 & -.2 & 6.63 & -3.63 & 2.67 & 0.33 & 3.0 & 0.0 \\
\hline 5 & 6 & 36 & 6.0 & 0.0 & 6.0 & 0.0 & 6.0 & 0.0 & 6.0 & 0.0 \\
\hline 6 & & 72 & 6.0 & 0.0 & 6.0 & 0.0 & 6.0 & 0.0 & 6.0 & 0.0 \\
\hline 7 & & 144 & 6.1 & -.1 & 15.7 & -9.7 & 2.1 & 3.9 & 6.0 & 0.0 \\
\hline 8 & Sub. & Tot. & 6.03 & -.03 & 9.23 & -3.23 & 2.7 & 3.3 & 6.0 & 0.0 \\
\hline 9 & 12 & 36 & 12.0 & 0.0 & 12.0 & 0.0 & 12.0 & 0.0 & 12.0 & .0 \\
\hline 10 & & 72 & 12.0 & 0.0 & 12.0 & 0.0 & 12.2 & -.2 & 12.0 & 0.0 \\
\hline 11 & & 144 & 12.0 & 0.0 & 20.4 & -8.4 & 4.0 & 8.0 & 12.1 & -.1 \\
\hline 12 & Sub. & Tot. & 12.0 & 0.0 & 14.8 & -2.8 & 9.33 & 2.67 & 12.03 & -0.03 \\
\hline 13 & Tot. & & -- & -.08 & ---- & -3.23 & ---- & 2.1 & ---- & -.01 \\
\hline
\end{tabular}




\section{Table 8}

Mean number of components retained and mean difference from population value for each level of number of variables $(p)$ and components $(m)$. Sample size $=450$. Component saturation $=.80$.

\begin{tabular}{|c|c|c|c|c|c|c|c|c|c|c|}
\hline \multirow[t]{2}{*}{ Row } & \multirow{2}{*}{$\begin{array}{l}\# \\
\text { m }\end{array}$} & \multirow{2}{*}{$\begin{array}{l}\# \\
p\end{array}$} & \multicolumn{2}{|c|}{ MAP } & \multicolumn{2}{|c|}{ K1 } & \multicolumn{2}{|c|}{ BART } & \multicolumn{2}{|c|}{ SCREE } \\
\hline & & & $x$ & $d$ & $x$ & $d$ & $x$ & d & $x$ & d \\
\hline 1 & 3 & 36 & 3.0 & 0.0 & 3.0 & 0.0 & 3.0 & 0.0 & 3.0 & -.3 \\
\hline 2 & & 72 & 3.0 & 0.0 & 3.0 & 0.0 & 3.0 & 0.0 & 3.0 & 0.0 \\
\hline 3 & & 144 & 3.0 & 0.0 & 3.0 & 0.0 & 3.0 & 0.0 & 3.0 & 0.0 \\
\hline 4 & Sub. & Tot. & 3.0 & 0.0 & 3.0 & 0.0 & 3.0 & 0.0 & 3.1 & -.1 \\
\hline 5 & 6 & 36 & 6.0 & 0.0 & 6.0 & 0.0 & 6.1 & -.1 & 6.0 & 0.0 \\
\hline 6 & & 72 & 6.0 & 0.0 & 6.0 & 0.0 & 6.2 & -.2 & 5.7 & 0.3 \\
\hline 7 & & 144 & 6.0 & 0.0 & 6.0 & 0.0 & 5.9 & 0.1 & 5.85 & 0.15 \\
\hline 8 & Sub. & Tot. & 6.0 & 0.0 & 6.0 & 0.0 & 6.07 & -.07 & 5.85 & 0.15 \\
\hline 9 & 12 & 36 & 12.0 & 0.0 & 12.0 & 0.0 & 12.3 & -.3 & 12.0 & 0.0 \\
\hline 10 & & 72 & 12.0 & 0.0 & 12.0 & 0.0 & 12.2 & -.2 & 12.0 & 0.0 \\
\hline 11 & & 144 & 12.0 & 0.0 & 14.0 & -2.0 & 11.0 & 1.0 & 12.1 & -.1 \\
\hline 12 & Sub. & Tot. & 12.0 & 0.0 & 12.67 & -.67 & 11.83 & 0.17 & 12.03 & -.03 \\
\hline 13 & Tot. & & --- & .00 & $-\cdots$ & -.22 & --- & 0.03 & --- & 0.01 \\
\hline
\end{tabular}


Table 9

Number and mean of over- and underestimations for each decision rule at both levels of "Component saturation".

Comp. Sat.

$$
\text { over }{ }^{\text {MAP }} \text { under over }{ }^{\text {K1 }} \text { under over }{ }^{\text {BART }} \text { under over under }
$$

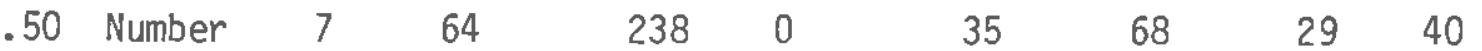

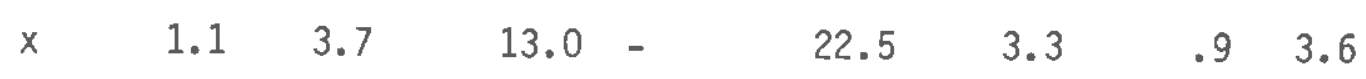

\begin{tabular}{|c|c|c|c|c|c|c|c|}
\hline 80 Number & 11 & 0 & 75 & 0 & 39 & 29 & 8 \\
\hline $\mathrm{x}$ & 1.0 & - & 5.4 & $=$ & 23.2 & 4.4 & 1.1 \\
\hline
\end{tabular}




\section{Table 10}

Percent correct specification of the number of population components

for each decision rule at both levels of "Components Saturation".

\begin{tabular}{|c|c|c|c|c|c|}
\hline Comp. S & & MAP & K1 & BART & SCREE \\
\hline .50 & $\%$ correct & $70.4 \%$ & $0.8 \%$ & $57.1 \%$ & $71.2 \%$ \\
\hline .80 & $\%$ correct & $95.4 \%$ & $68.8 \%$ & $71.7 \%$ & $96.7 \%$ \\
\hline Overall & $\%$ correct & $82.9 \%$ & $34.8 \%$ & $64.4 \%$ & $84.0 \%$ \\
\hline
\end{tabular}


Table 9

Mean difference from population criterion at each level of "Component Saturation" and "Number of Components" for each decision rule.

MAP

d

Comp: \#

Sat. Comp.

.50

$\begin{array}{lr}3 & -.075 \\ 6 & 0.288 \\ 12 & 5.388 \\ & .1\end{array}$

.1

Printout cancelled by operator

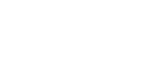

K1

d
BART

d

SCREE

d

$-20.112$

$-2.762$

$-0.025$

$-18.362$

$-3.75$

$-0.025$

$-14.469$

0.312

1.688

$-1.925$

$-4.488$

$-0.038$ 